\title{
Idiopathic Hypereosinophilic Syndrome/Eosinophilic Leukaemia (Case Report): A Challenge to Diagnose
}

\author{
Sathyavathi Alva, Angshuman Saha, Tahera Syed, T. M. Kariappa \\ Department of Pathology, K. V. G. Medical College, Sullia, D. K, Karnataka, India
}

Email address:

angshuman.saha3@gmail.com (A. Saha)

\section{To cite this article:}

Sathyavathi Alva, Angshuman Saha, Tahera Syed, T. M. Kariappa. Idiopathic Hypereosinophilic Syndrome/Eosinophilic Leukaemia (Case Report): A Challenge to Diagnose. Science Journal of Clinical Medicine. Special Issue: Latest Different Concepts of Gynaecology. Vol. 4, No. 4-1, 2015, pp. 16-18. doi: 10.11648/j.sjcm.s.2015040401.14

\begin{abstract}
Hypereosinophilic syndrome (HES) was first described by Hardy and Anderson in 1968 . HES may result either from eosinophilic differentiation of a clone of neoplastic cells or from reactive eosinophilia. It appears lightly that in many patients idiopathic HES is actually a chronic myeloproliferative disorder. There is vaguely overlapping clinico-pathological picture of HES with (Chronic eosinophilic leukaemia) CEL which often adds to diagnostic confusion. In 2001, the World Health Organization (WHO) proposed a set of criteria that distinguish CEL from HES. An evidence of genetic clonality of eosinophils or an increase in blast cells in blood or bone marrow is mandatory for diagnosis of CEL, while no specific diagnostic tests exists for HES, making it an entity of exclusion. A 36 year old female presented with intermittent fever and dry cough since two months. Clinical examination revealed mild pallor with hepatosplenomegaly. Chest examination showed bilateral basal crepitations. The patient was subjected for hematological, biochemical and radiological assessment. The peripheral smear and bone marrow aspirates revealed presence of eosinophilic precursors with predominance of eosinophiloblasts associated with eosinophilic myelocytes, metamyelocytes, a few myeloblasts and lymphocytes, the features of which are in favour of CEL. CEL, a rare myeloproliferative entity and its presentation with pulmonary manifestations is still rare in India. Hence, we present this rare entity along with review of available literature.
\end{abstract}

Keywords: Chronic Eosinophilic Leukaemia, Hypereosinophilic Syndrome, Pulmonary Manifestations

\section{Introduction}

Hypereosinophilic syndrome (HES) was first described by Hardy and Anderson, but the diagnostic criteria of this hematological disorder were proposed by Chusid et al. in $1975^{8}$ and have been almost invariably accepted until recently.

These criteria include unexplained severe peripheral blood eosinophilia (higher than 1500 eosinophils $/ \mathrm{mm}^{3}$ ) sustained for over 6 months and accompanied by end-organ damage resulting from direct organ infiltration by eosinophils. The generic term "unexplained eosinophilia" has been used to exclude any allergic, inflammatory, infectious or neoplastic diseases, including specific eosinophilia induced by chronic or acute myelogenous leukemia, myelodysplastic syndromes or other myeloproliferative disorders.

In 2001, the World Health Organization (WHO) proposed a set of criteria that distinguish CEL from HES ${ }^{9}$. These criteria were based on the exclusion of the diseases mentioned above, together with the absence of a T-cell population with an aberrant phenotype and abnormal cytokine production, and the presence of a clonal cytogenetic abnormality or clonality, or a blast content in the peripheral blood (higher than 2\%) or marrow (more than $5 \%$ but less than $19 \%$ ).

Two types of HES/CEL, in particular have been described; Intrinsic: those with clonal expansion of a myeloid progenitor population with primary eosinophil differentiation including FIP1L1/PDGFR $\alpha+$ CEL and CEL demonstrating with cytogenetic abnormalities; and Extrinsic: eosinophil expansion responding to a clonal expansion of Tcells, expressing high levels of the eosinophildifferentiating cytokine interleukin-5 (IL-5), including a subgroup of HES/CEL patients who present clonal T-cell populations, expressing aberrant phenotypes and producing 
Th2 cytokines such as IL- $5^{2}$.

HES may result either from eosinophilic differentiation of a clone of neoplastic cells or from reactive eosinophilia. In other patients HES is idiopathic. It appears lightly that in many patients idiopathic HES is actually a chronic myeloproliferative disorder. Those cases showing an increase of blast cells or a demonstrable clonal cytogenetic abnormality should be classified as eosinophilic leukemia ${ }^{3}$.

According to available data, the incidence is highest in the fourth decade of life and the disease most commonly affects males. In CEL the eosinophilic count is greater than or equal to $1.5 \times 10^{9} / \mathrm{L}$. The rarity of CEL is further compounded by a variety of other disease processes accompanied by chronically persistent eosinophilia ${ }^{4}$.

Here we present this rare entity which presented clinically with vague signs and symptoms of pulmonary infection.

\section{Case Report}

A 36 year old female presented with intermittent fever and dry cough since two months. She did not reveal any obvious history of smoking, alcohol or drug abuse, any allergic disease and no exposure to any toxins/pesticides. On examination she was febrile with a temperature of $100.5^{\circ} \mathrm{F}$, pulse rate was 110 beats/minute with normal rhythm. Clinical examination revealed mild pallor with hepatosplenomegaly. Chest examination showed bilateral basal crepitations. The patient was subjected for hematological, biochemical and radiological assessment.

Hematological investigations revealed Hemoglobin $10.6 \mathrm{gm} / \mathrm{dl}$, Total leucocyte count -59000 cells/cu mm with an Absolute eosinophil count of $53,100 \mathrm{cells} / \mathrm{cu} \mathrm{mm}$, Platelet count $-90,000$ cells $/ \mathrm{cu} \mathrm{mm}$. The differential count showed abnormally increased eosinophils (90\%), myeloblasts $(4 \%)$, myelocytes $(2 \%)$, metamyelocytes $(2 \%)$, neutrophils $(1 \%)$ and lymphocytes $(1 \%)$. The reticulocyte was $1 \%$.

Peripheral smear examination revealed mild microcytic hypochromic anemia with eosinophils and eosinophiloblast, few myeloblast, myelocytes and thrombocytes. (fig 1\&2)

Bone marrow aspirates were hypercellular dominated by eosinophilic precursors with striking presence of eosinophiloblasts, eosinophilic myelocytes, metamyelocytes, a few myeloblasts and lymphocytes, compromising erythroid and megakaryocytic elements.(fig3\&4)

Biochemical parameters were within normal limits.

Chest X Ray showed microcalcifications.

Stool examination was negative for parasitic ova, cysts or blood cells. Serology was negative for Hepatitis B and human immunodeficiency virus (HIV).

\section{Discussion}

An increase in the blood eosinophil count may occur in a number of diseased states including allergies, parasitic infections, vascular disease and as a reaction to the presence of malignant tumors ${ }^{5}$.

Presently, an unexplained chronic persistent eosinophilia with a demonstrable cytogenetic abnormality or an increase in blast cells is considered as eosinophilic leukemia ${ }^{7}$.

The modern diagnostic criteria as proposed by World Health Organization ${ }^{1}$ for CEL include:

1. Persistent eosinophilia $\geq 1.5 \times 109 / \mathrm{L}$ in blood, increased bone marrow eosinophils

2. $>5 \%$ but $<19 \%$ myeloblasts in the bone marrow or $>2 \%$ in the peripheral blood

3. Clonality of myeloid cells

4. No reactive eosinophilia due to allergy, parasitic, infectious, pulmonary, or collagen vascular disease

5. No reactive eosinophilia due to other malignancies:

- T-cell lymphomas

- Mastocytosis

- Acute lymphoblastic leukemia/lymphoma

- Hodgkin's lymphoma

- Other myeloproliferative diseases

- Myelodysplastic syndrome

- Acute myeloid leukemia including inv (16), t (16; 16)

- $\mathrm{CML}$

6. No T-cell population with abnormal cytokine production and aberrant phenotype

Differential diagnosis of eosinophilia ${ }^{6}$

Any patient diagnosed with eosinophilia (eosinophils .0 .5 $\left.\mathrm{x} 10^{9} / 1\right)$, falls into one of the three categories of disease described as follows:

(i) Reactive eosinophilia

(ii) Clonal eosinophilia

(iii) Unexplained (idiopathic) eosinophilia

Our understanding of and treatment options for patients with eosinophilic leukaemia and the idiopathic hypereosinophilic syndrome have improved greatly in recent years, the major advance being the discovery of the cytogenetically silent FIP1L1-PDGFRA fusion and the response of the associated syndrome to imatinib therapy ${ }^{6}$.

In our case, chronic eosinophilic leukaemia as a presumptive diagnosis was made based on the morphologic features of peripheral blood smear and bone marrow aspiration study, though the karyotype could not be investigated. Referral to another centre for cytogenetic analysis and further evaluation and management was declined for financial constraints.

Our case study is an attempt to highlight the need for caution in diagnosing patients with unexplained eosinophilia. 

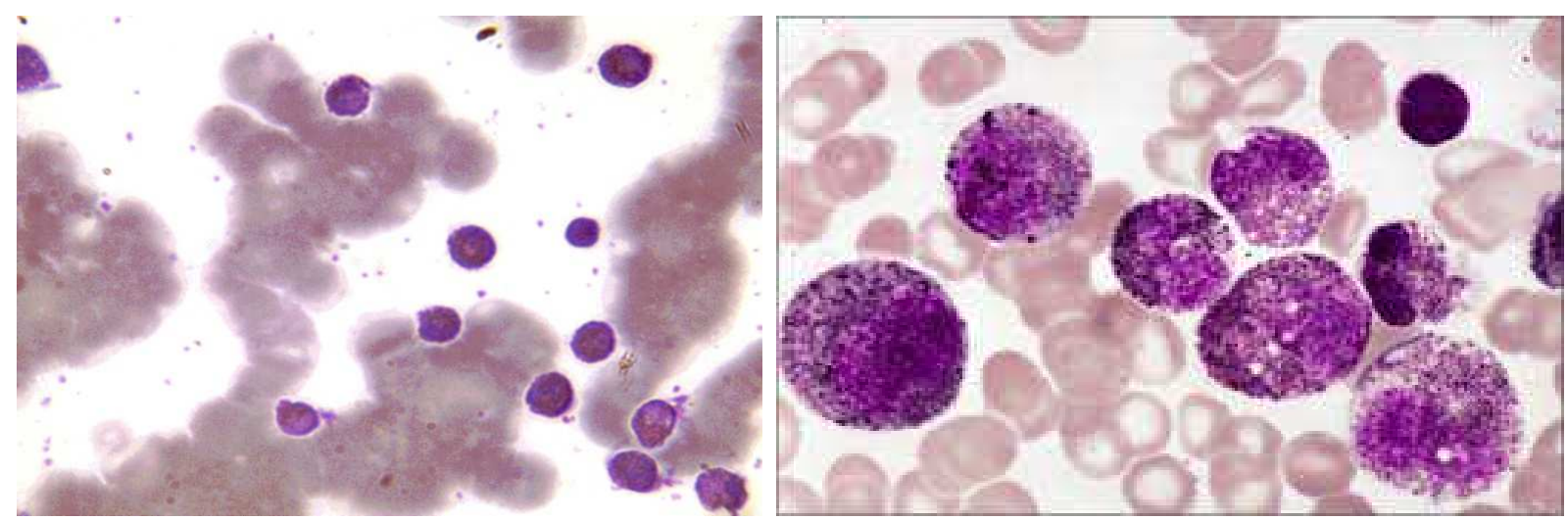

Figure 1 \& 2. Peripheral blood smear showing dysplastic eosinophils ( 40 objective and $x 100$ oil immersion objective).
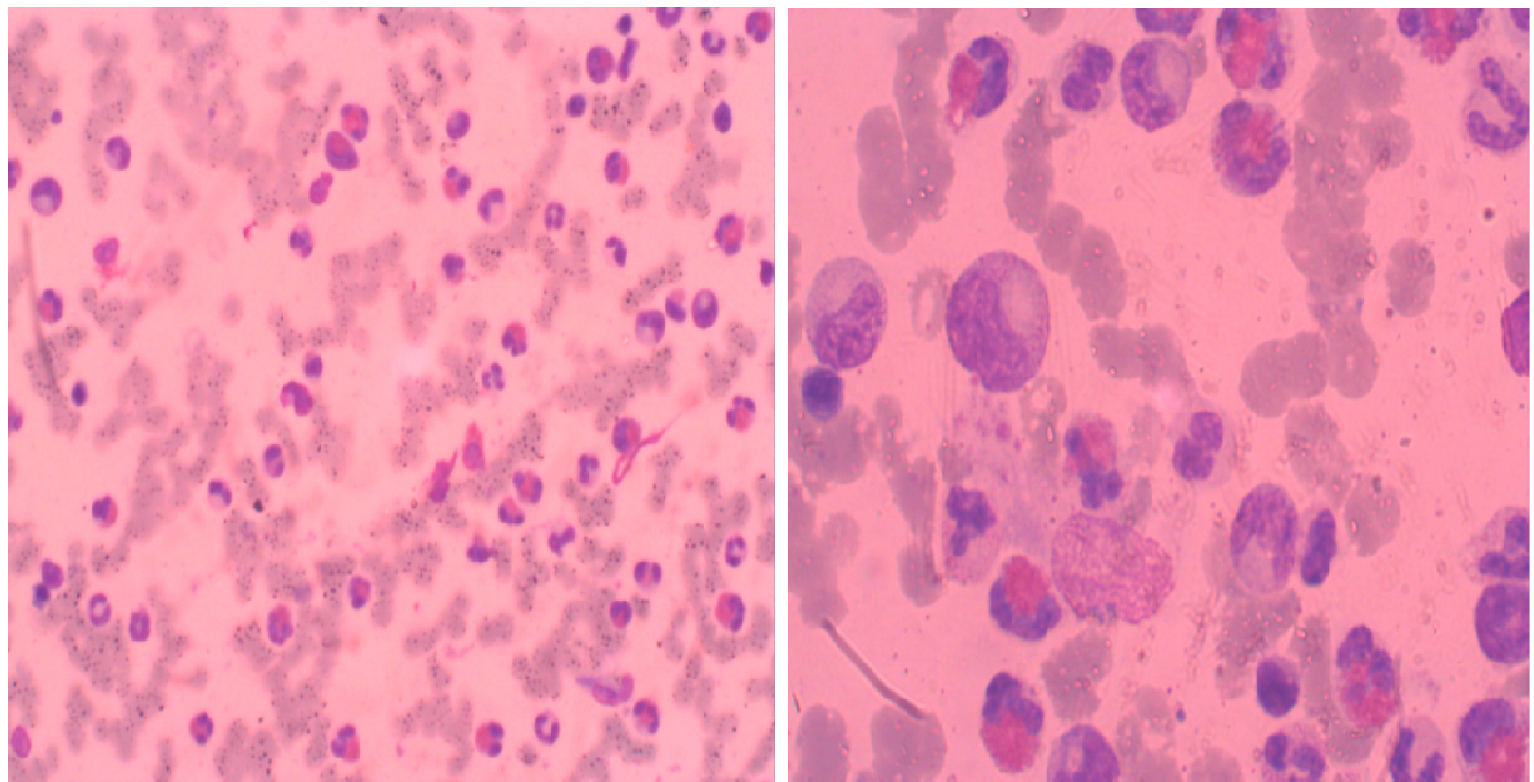

Figure 3 \& 4. Bone marrow aspirate showing eosinophiloblast, eosinophilic myelocytes, metamyelocytes and a few myeloblasts. $(x 40$ objective and $x 100$ oil immersion objective).

\section{References}

[1] Vardiman JW et al Chronic myeloproliferative diseasesand Myelodysplastic / myeloproliferative diseases. World Health Organization Classificationof Tumours. Pathology and Genetics.Tumours of Haematopoietic and Lymphoid Tumours. H. N. Jaffe ES, Stein H, Vardiman JW. Lyon, France, IARC Press: 2001, 17-31, 47-52.

[2] Yamada Y, Rothenberg M.E, Cancelas J.A. Current concepts on the pathogenesis of the hypereosinophilic syndrome/chronic eosinophilic leukaemia. Translational Oncogenomics 2006:1

[3] Bain B.J. British journal Hematol 1996 ;95,2-9

[4] Babu K.V.S, Chowhan A.K, Rukmangadha N, Vengamma B,
Reddy M.K. Chronic eosinophilic leukaemia : a case report. J Clin Sci Res 2012; $1: 46-8$

[5] Brito-Babapulle F. Blood Rev, 1997 ; 11,129-45

[6] Fletcher S and Bain B. Eosinophilic Leukaemia. British Medical Bulletin 2007; 81 \& 82:115-127

[7] Kumar A, Sinha S, Tripathi A.K. Chronic Eosinophilic Leukaemia: a case report and review of literature. Indian J Hematol, Blood Transfusion.2014, 23:112-115

[8] Chusid, M.J., Dale, D.C., West, B.C. and Wolff, S.M.. The hypereosinophilic syndrome: analysis of fourteen cases with review of the literature. Medicine (Baltimore) 1975 54:1-27.

[9] Bain, B., Pierre, R., Imbert, M., Vardiman, J.W., Brunning, R.D. andFlandrin, G.2001 Chronic eosinophilic leukaemia and the hypereosinophilic syndrome. IARC Press, Lyon, France. 